\title{
Model-integrated Tools for the Design of Dynamically Reconfigurable Systems
}

\author{
TED BAPTY ${ }^{\mathrm{a}, *}$, SANDEEP NEEMA $^{\mathrm{a}}$, JASON SCOTT $^{\mathrm{a}}$, \\ JANOS SZTIPANOVITS $^{\mathrm{a}}$ and SAMEH ASAAD \\ ${ }^{\mathrm{a}}$ Institute for Software Integrated Systems, Vanderbilt University; ${ }^{\mathrm{b}}$ IBM T.J. Watson Research Center
}

(Received 1 February 1999; In final form 1 October 1999)

\begin{abstract}
Several classes of modern applications demand very high performance from systems with minimal resources. These applications must also be flexible to operate in a rapidly changing environment. Achieving high performance from limited resources demands application-specific architectures, while flexibility requires architectural adaptation capabilities. Reconfigurable computing devices promise to meet both needs. While these devices are currently available, the issue of how to design these systems is unresolved. This paper describes an environment for design capture, analysis and synthesis of dynamically adaptive computing applications. The representation methodology is captured in a Domain-Specific, Model-Integrated Computing framework. Formal analysis tools are integrated into the design flow to analyze the design space to produce a constrained set of solutions. HW/SW Co-simulations verify the function of the system prior to implementation. Finally, a set of hardware and software subsystems are synthesized to implement the multi-modal, dynamically adaptive application. The application executes under a runtime environment, which supports common execution semantics across software and hardware. An application example is presented.
\end{abstract}

Keywords: Reconfigurable computing, FPGA, HW/SW co-design, HW/SW synthesis, FPGA, $\mathrm{HW} / \mathrm{SW}$ co-simulation, dynamic reconfiguration, design environment, Model-Integrated Computing

\section{INTRODUCTION}

Modern high-performance embedded systems, such as Automatic Target Recognition for Missiles or Dynamic Protocols Mobile Communications devices, face many challenges. Power and volume constraints limit hardware size. Accurate, high-performance algorithms involve massive computations. Systems must respond to demanding real-time specifications. In the past, custom application-specific architectures have been used to satisfy these demands.

*Address for correspondence: 400 24th Ave. S., Nashville, TN 37235. Tel.: 615-343-6709, Fax: 615-343-6702, e-mail: bapty@, vuse.vanderbilt.edu 
This implementation approach, while effective, is expensive and relatively inflexible. As the world demands flexible, agile systems, the hardwired application-specific architectures fail to meet requirements and become expensive to evolve and maintain. As new algorithms are developed and new hardware components become available, a fixed, application specific architecture will require significant redesign to assimilate the technologies.

Flexible systems must function in rapidly changing environments, resulting in multiple modes of operation. On the other hand, efficient hardware architectures must match algorithms to maximize performance and minimize resources. Structurally adaptive, reconfigurable architectures can meet both these needs, achieving high performance with changing algorithms. Reconfigurable computing devices, such as Field Programmable Gate Arrays allow the implementation of architectures that change in response to the changing environment.

The field of Reconfigurable Computing is rapidly advancing for scientific and Digital Signal Processing applications [1 -3]. While today's Field Programmable Gate Array technology shows great promise for implementing reconfigurable computational systems, their capabilities in certain areas (such as floating point arithmetic) cannot equal other technologies. For this reason, efficient system architectures must encompass a heterogeneous mix of the most suitable technologies, along with the capability to dynamically restructure the system architecture. The target systems are built on a heterogeneous computing platform: including configurable hardware for computation and structural adaptation, and ASIC's, generalpurpose processors and DSPs for computation. The primary difficulty in this approach lies in system design. A designer must now maintain a set of diverse system architectures, which exist at different times in the system's lifetime, and map these architectures onto the same group of resources. The designers must manage the behavior of the system, determining the operational modes of the system, the rules for transitioning between operational modes, and the functional properties within each operational mode. In addition, the system must make efficient use of the resources, enabling the designer to minimize the envelope of hardware required to support the union of all operational modes. Current system design tools are insufficient to manage this complexity.

\section{State of the Art}

The standard methods for the design of hardware systems use VHDL specifications with use of offthe-shelf components, such as libraries of parameterized modules (LPM). This approach allows hierarchical design of large systems of a fixed structure. The ability to specify multiple behavioral designs for a single entity allows a complex design space to be created. The choice of configuration of a system is largely a manual process. Using this approach to design a dynamically reconfigurable system would involve multiple designs, with only a manual linkage between the individual design modes. Custom runtime support would be added to manage the reconfiguration. This approach relies too much on manual, informal interaction between modes. The pure VHDL approach also provides little support for software/ hardware interaction.

The Ptolemy design environment [4] has recently been extended to support reconfigurable hardware. Ptolemy is a comprehensive package that supports hardware/software codesign and heterogeneous architectures, spanning from microprocessors/DSP's to FPGA's to MEMS devices. It is unclear about the support for dynamic reconfiguration design and runtime support.

DEFACTO from USC/ISI [5] is a high-level design tool for design and synthesis of reconfigurable systems. In DEFACTO algorithms are specified in a high-level programming language such as C or MATLAB. The target architecture is specified in an architecture description language. The target architecture is assumed to be consisting of a general-purpose processor (GPP) and a number of configurable computing units (CCU). A parallelizing compiler partitions computation 
and control among the GPP and the CCUs, and also manages data storage and communications. The primary drawback of this approach is in the use of sequential programming languages in system specification. The effectiveness and scalability of programming languages in specifying large, complex systems with parallel interacting components is not determined. Runtime support for dynamic reconfiguration has not been addressed. MATCH from NWU [6] employs a similar approach, however, the system specifications are solely in MATLAB.

The DISC project at BYU [7] employs dynamic reconfiguration to support demand-driven instruction set modification. It can partially reconfigure an FPGA device to page custom applicationspecific instructions in and out of the device. on demand. While the approach takes advantage of the partial reconfiguration of the instruction set to improve the functional density, it does not address the concerns in performing global system level reconfiguration.

The CHAMPION project at UTK [8] uses KHOROS, a popular design tool for Image and Signal processing applications, to capture system specifications. These specifications are then automatically transformed, partitioned, and mapped to a distributed, heterogeneous resource network, consisting of DSPs and FPGAs. The objective of the partitioning process is to partition not only in space but also in time. However, it is not clear as to how the reconfiguration process will be orchestrated at runtime.

Several design approaches exist for low-level dynamic reconfiguration. Some of these involve unique FPGA designs with multiple contexts allowing rapid switching between a limited number of unique designs. Tools for high-level system design using these components in a heterogeneous system have not yet appeared.

\section{Target of this Paper}

High-level design tools are being developed to capture designs and to generate functional systems as part of the DARPA Adaptive Computing Systems Program. This paper describes a modelintegrated approach to be used in the development of reconfigurable systems. There are many significant issues in the development process. The approach described here divides these issues into several categories: (1) Representation and Capture of design information in terms of Models; (2) Analysis of the models for design/requirements/ resource trade-off studies; (3) Synthesis of architectures and executable systems directly from the models; and (4) Runtime support environments to support efficient execution of the synthesized reconfigurable systems.

The Model-Integrated Computing (MIC) approach has been successfully applied to a diverse set of applications [9-14]. The general MIC approach involves creating a development environment that is customized for a specific application domain. The resultant development environment is a multiple-aspect graphical editor that directly supports the engineering concepts required in the development process. Where several engineering disciplines are involved in system development (e.g., Software, Hardware, DSP algorithms, Systems Requirement Specification, etc.), the multiple-aspect nature of the approach allows different aspects to be customized for individual disciplines. The graphical editor allows construction of system Models, which capture the specifications and components required along with their relationships. The Models form a database of design information that can then be used in system analysis, trade-off studies, and performance estimation/simulation. These same Models are used to synthesize the executing systems. The synthesis process assumes a runtime environment that hides the low-level hardware/ software details from the synthesis process.

This paper will follow a logical progression in describing the Model-Integrated Computing approach for adaptive systems design. The first section will describe the rationale and implementation of the design capture approach. The next section will give an overview of the current and 
planned analysis capabilities for design-space exploration. The following sections will describe the system synthesis process and the runtime environment architecture and implementation. Finally, we will show the implementation of a missile Automatic Target Recognition application incorporating adaptive system behavior.

\section{DESIGN REPRESENTATION}

The customization of the Model-Integrated Computing design environment involves a careful analysis of the needs of the design engineers, the methods and components used in the designs, and the target systems. For an environment to successfully support the creation of systems, the concepts used by designers must be faithfully reproduced by the design environment. The critical concepts required for dynamically adaptive computing architecture targets include the ability to specify the dynamic behavior of the system (in terms of Modes), the function of the system in each of these modes (inputs, outputs, and algorithms), and the resources available. Each of these concepts can be modified by constraints known to the designer. Interactions between the concepts are captured via references within the models. The integration of multiple modeling concepts with the constraints and references are critical in the control and optimization of the dynamically adaptive behavior of the target system.

This section will describe the concepts developed in the creation of the Adaptive Computing Systems MIC environment.

The Adaptive Computing Systems (ACS) environment divides the design process into four major categories:

1. Behavioral Modeling Dynamically adaptive systems must manage multiple system behaviors. In this first category, the adaptive behavior is defined. The designer can specify the operating modes of the system, the legal transitions between modes (and the conditions for transition), and the specifications for system operation while in each operating mode.

2. Algorithm/Structural Modeling In this category, potential algorithms are described. The algorithms define signal flow specifications to compute required system outputs.

3. Resource Modeling The resource models describe the hardware available for construction of the system. This consists of physical processors, devices, and the interconnection topology.

4. Constraint Specification These modeling categories are augmented and linked together with a Constraint framework. The Constraints allow user-defined interactions to be specified, establishing linkages between properties in one category and objects in the same or another category. This modeling category also allows linkages between processing modules in adjacent modes, to guide the transition of state information between computational structures.

\section{Behavioral Models}

Behavioral models capture the dynamic behavior of the system and the potential interactions between modes of operation Since the system will be operating in discrete modes, with specific transitions between these modes, a Discrete Finite State Machine formalism is used [15] (Fig. 1). States define operational modes of the system. Transitions define the potential conditions required for the system to change modes and the end-state of the mode-shift. In order to manage system complexity, where the system may have many potential operational modes, states have hierarchical decomposition.

The event expression that triggers a mode change is defined by the transition rules. A transition rule is a Boolean equation composed of event variables. When the transition rule expression is satisfied, system reconfiguration occurs. Inputs to the transition rules, event variables, are computed in the Algorithmic/Structural modeling view described below. Event variables can 


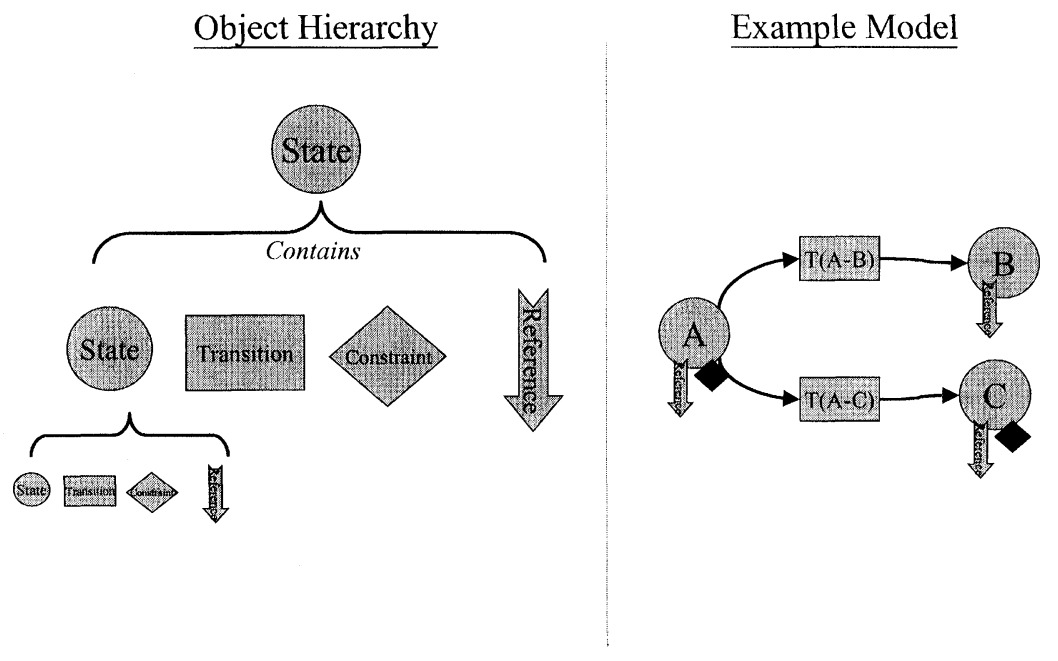

FIGURE 1 Behavioral model.

be directly sampled external signals or complex computational results.

The behavioral modeling aspect is linked to the Algorithmic/Structural aspect by References. A Reference allows the user to establish a pointer from the mode to a defined computational algorithm. Each mode references a model in the Structural Aspect that defines the processing algorithm that is to be operational in that mode. The references allow a single algorithm to be applied to any number of system states.

The behavioral modeling aspect also allows the specification of constraints, such as real-time requirements and maximal runtime power usage. Maximal permitted system delays can be specified for any pair of input and output ports on the algorithm model. Maximum power limits are specified using attributes of the models. Constraints capture the system performance requirements.

\section{Algorithm/Structural Models}

The structural modeling aspect is used to define the processing algorithm structure. Algorithms are described in terms of computational components and data interactions. To manage system complexity, a multi-level hierarchy is used to structure algorithm definition.
The algorithm is modeled as a dataflow structure with the three classes of objects: compounds, primitives, and templates. The relationship between these objects is captured in Figure 2.

A primitive is the basic element, representing a numerical processing operation. A primitive maps directly to an implementation in either hardware or software. Primitive objects are annotated with attributes, which capture measured performance, resource (memory) requirements, and other userdefined properties.

A compound is an aggregation object that contains primitives, other compounds, and/or templates. These components can be connected within the compound to define the algorithmic dataflow. Compounds provide the hierarchy in the structural description that is necessary for managing the complexity of large designs.

A Template object captures a design alternative. The Template allows the specification of multiple algorithm architecture alternatives for a given task. These design alternatives can be composed of Compounds, Primitives, or other Templates, allowing hierarchies of design alternatives. The selection among the alternatives occurs at model interpretation, allowing design flexibility to be specified.

Design alternatives allow the model of the system to capture a range of design possibilities. 


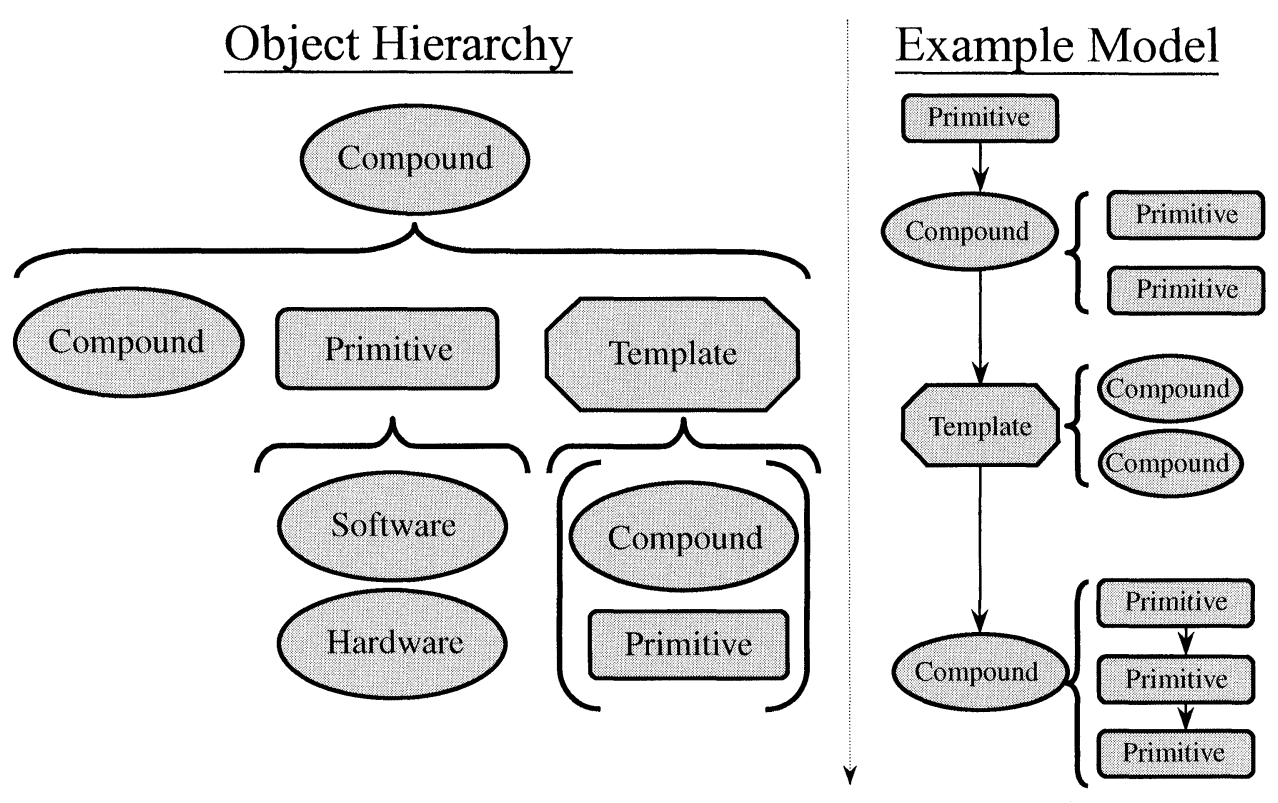

FIGURE 2 Compound/primitive/template structure.

The large design space gives the tools the freedom to search for and select an implementation that meets the specified requirements and fits within available resources. Each of these alternative methods has different performance attributes and different hardware requirements. The selection of the best alternative depends not only on the hardware that is available, but also on whether the hardware is to be time-shared, and what hardware is already allocated to support the processing algorithms that are required for operations in different modes.

For the high-level designer, algorithm alternatives allow a virtual separation of algorithm from implementation. Typical algorithm design requires the engineer/physicist to consider the hardware details of the underlying architecture to achieve an efficient implementation. The ultimate effect is that the resulting algorithm reflects the hardware structure. This practice leads to highly nonportable, technology-specific designs. System upgrades to use more modern technology require a bottom-to-top redesign. Algorithm alternatives separate the algorithm from the architecture, to postpone the implementation decisions to a much later step in the design process, simplifying technology migration efforts.

\section{Resource Models}

Resource models define the target hardware platform. Resources are modeled in terms of hardware components and the physical connections among them. The relationships among the resource model components are shown in Figure 3.

The top-level hardware system is a Network of components. Network components are either programmable processor elements (such as DSPs or standard RISC/CISC processors), programmable logic components (such as FPGAs), or dedicated hardware ASIC components for fixed functions (such as FFT computation). Data Sources and Data Sinks capture the specifics of hardware $\mathrm{I} / \mathrm{O}$ interfaces and data acquisition/effector interfaces.

The components are constructed using cores and ports. Every processing element must contain one core. The core object captures the inherent 


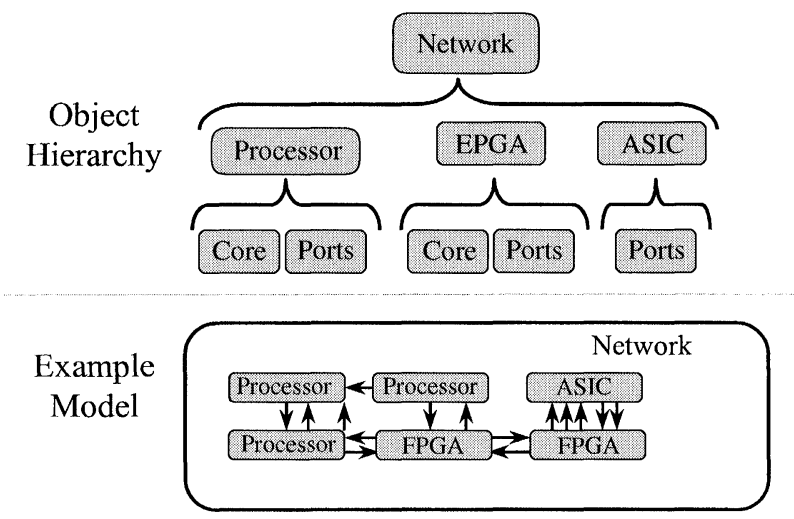

FIGURE 3 Resource model.

performance attributes of the processing element such as clock speed, memory, and other resources. A port represents a physical communication channel. Ports have associated protocols and specific pin assignments, capturing physical connection points on a chip. Connections between processing elements are created by connections between ports. The connections capture the "as-built" topology of the physical implementation.

\section{Constraint Specification}

System constraint specifications have four categories of design constraints: (a) operational constraints, (b) composability constraints, (c) resource constraints, and (d) performance constraints. These constraints establish linkages between modeling object properties in different modeling categories.

Operational constraints express conditions relating design configurations to operational modes. These constraints are applied within the Behavioral models. Operational constraints can be used to restrict implementation alternatives based upon current operational mode.

Composability constraints are logic expressions that restrict the composition of alternative processing blocks. These constraints express compatibility between related implementation options. For instance, if hardware FFT is selected from different alternatives in a processing block, then the hardware IFFT must be selected in a related processing block.

Resource constraints are logic expressions describing the selection of processing blocks based on resource limitations. These constraints allow hardware requirements to be specified for software components. For example, a software component may require access to a large block of memory or to a hardware unit.

Performance constraints are integer constraint expressions governing the end-to-end latency, throughput, power consumption, and/or space/ volume. Some of the performance constraints are implicitly specified in the properties of Behavioral models. These constraints allow the designer to control the potential design space for the analysis/ synthesis process and to ensure that the synthesized system satisfies real-time constraints.

\section{MODEL ANALYSIS}

A design described in the modeling environment defines a design space consisting of modes and requirements, potential implementations, and resource sets. The designer must select appropriate combinations of implementations and resource assignments for all of the desired operational modes. Given the flexibility in defining design 
alternatives, this space can be extremely large (moderately sized design examples have defined a space of $10^{24 t h}$ ). A designer cannot handle such a large design space without sufficient tools. The design space must be evaluated to find a set of designs (mode configuration pairs) that best satisfy the design criteria.

There are a large number of conflicting design criteria in reconfigurable systems. These criteria must be applied across all of the system operational modes. The processing needs of each of the system modes must be satisfied with a single shared hardware platform. The analysis tools must allow efficient exploration, navigation, and pruning of this space to select feasible hardware/ software architectures for user-definable cost functions such as weight, power, algorithmic accuracy and flexibility. Given the size of the design space, and the complexity of the analysis, a powerful, scalable analytical method is required.

\section{Constraint Satisfaction Using Symbolic Methods}

The design space exploration tool uses a symbolic method based on Ordered Binary Decision Diagrams to represent, navigate, and prune the design space. In this symbolic representation, sets/spaces are represented as a Boolean expression over the members of the set. The members of the set are encoded as binary variables under a binary encoding scheme. The principal benefit of the approach is that it does not require enumeration of the set/space to perform operations.

Ordered Binary Decision Diagrams [16, 17] are a canonical representation of logic functions, representing Boolean functions as directed acyclic graph in a memory-efficient format. The operations over the Boolean functions are implemented as graph algorithms, thereby rendering "manipulation" of the space fast and efficient.

With this symbolic formalism, the application of logical constraints is relatively straightforward. The user-defined logical constraints can be represented as a Boolean expression over the components of the design space. Constraint application is a conjunction of the constraint Boolean expression with the Boolean expression that represents the design space. The resultant Boolean expression represents the "constrained" design space. Application of the integer arithmetic constraints such as timing and power constraints requires further analysis (see [20] for details), however the basic approach remains the same.

While the approach scales well, in very large design spaces with many constraints applied an exponential explosion of the OBDD's can occur. To address this problem we support hierarchical constraint processing. The constraint processing is done hierarchically with constraints scoped to a particular level; i.e., constraints are applied to sub-spaces first, pruning them to the extent possible and then progressing upwards in the hierarchy. This technique is very effective when there are a large number of constraints with a limited scope. The technique is not effective when there are many globally scoped constraints in a large design space.

The constraints "prune" the design space by enforcing the requirements specified in the constraint. These constraints can be iteratively applied to the design space, with the goal of reducing the

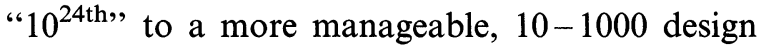
alternatives. We have implemented the approach described above in a design space exploration tool. Design engineers can iteratively apply constraints and visualize the sensitivity of the design space to the constraint. If a constraint is extremely tight, its application can eliminate the design space altogether. In this case, the constraint can be released and other constraints can be applied instead. The outcome of the constraint satisfaction step is a set of design configurations much smaller than the original design space. Figure 4 shows a screen capture of the design space exploration tool. When the design engineer is satisfied with this constrained design set, the design process continues with simulation. 


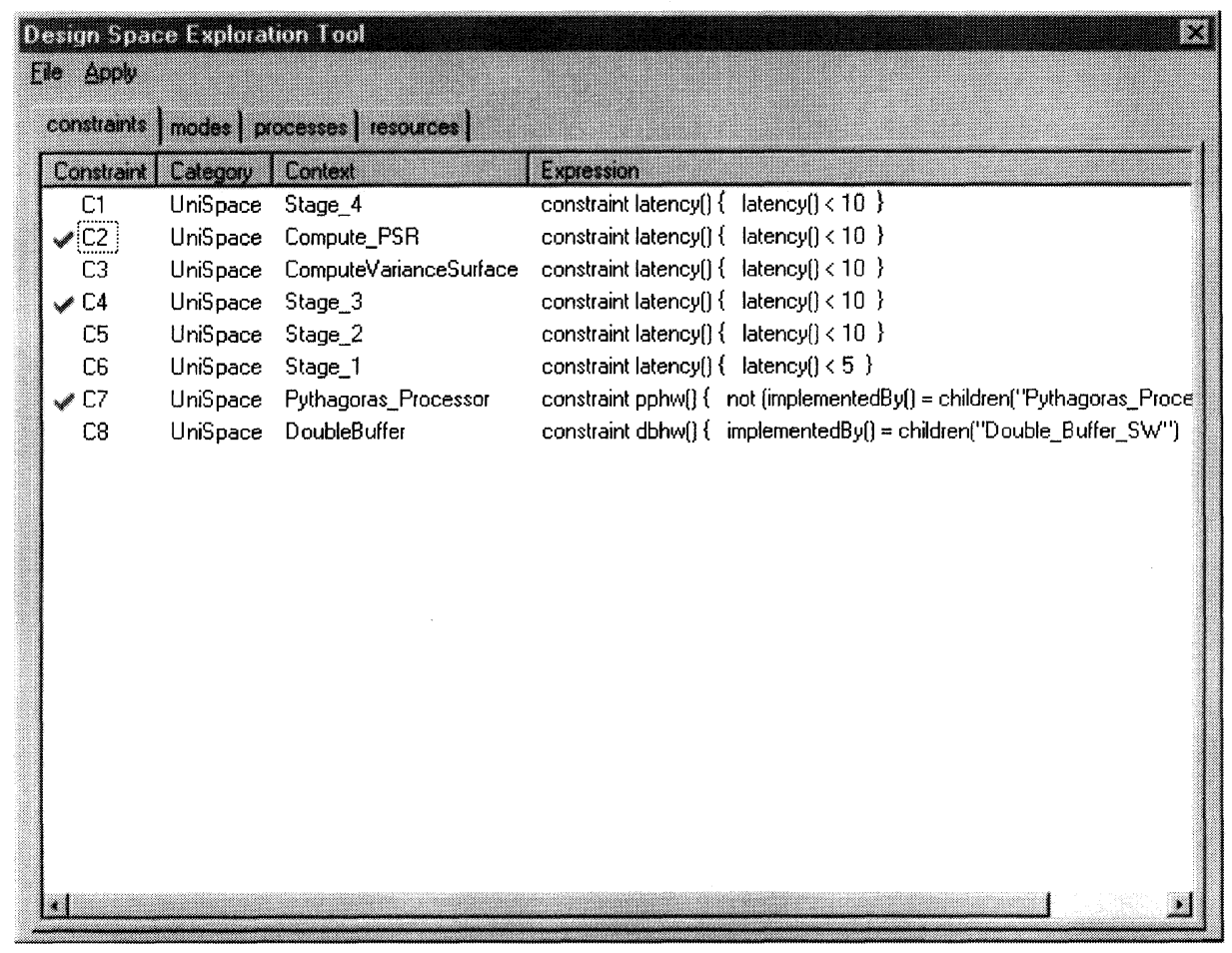

FIGURE 4 Design space exploration tool.

\section{HW/SW Co-simulation}

The model constraints encode the behavior of the system with a relatively high level of granularity. While a large granularity is necessary to work with tremendously large design spaces, the precision of this approach can be too low. The designer will be required to accept designs that are near the fringes of the constraint envelope, due to constraint uncertainty. To establish a more accurate estimate of in-system performance, a simulation capability is required. Since the target of the tool is hardware and software, the simulator must support co-simulation.

While this research is still at its early phases, the current approach is to allow the system designer to perform co-simulation at three levels of abstraction. These levels provide a trade-off between execution speed and accuracy of results. Simulation can occur at the performance level, the algorithm level and the gate/instruction level. This will enable the designer to quickly "zoom-in" on the more viable design alternatives and perform more accurate simulations only on this subset.

To be useful for rapid design space exploration, the co-simulation environment must be seamlessly integrated with the rest of the system. Information used to automatically construct the simulation testbench at various levels is directly extracted from the model database to ensure consistency among various levels of detail. Different levels of simulations can be generated that use different, possibly overlapping subsets of the model database. Output from the simulation is interpreted and fed back in a high level form to the user in the same design environment. This is an important aid in the interpretation of the data. In addition to simulating each mode individually, the process of mode shifts must be simulated to estimate the cost of reconfiguration. 
At the performance level, only the performance of the structural model is simulated. Performance attributes, such as latency and throughput, associated with processing primitives are used to construct a network of delay models for the system. Data flow is represented by tokens for faster simulation via packages such as PML $[18,21]$. No distinction of hardware versus software implementation is made at this level, however different components will have different performance attributes. The output of this step will be an overall performance assessment of the proposed algorithm, flagging the critical components or hot spots of the system.

At the algorithm level, the functional computation is simulated. The simulation does not include low-level timing details, allowing the user to quickly verify the correct numerical functionality of the system. Hardware functions are described in VHDL and software functions are described in $\mathrm{C}$ and encapsulated in a VHDL-wrapper entity. A commercial VHDL simulator equipped with a foreign language interface will be the target for mapping.

The lowest level of abstraction is the gate/ instruction level co-simulation. At this level, a $\mathrm{HW} / \mathrm{SW}$ co-simulation environment is constructed that models the system platform as described in the resource models of Section 1. VHDL simulation models will be used to describe hardware components such as ASICs and FPGAs. Processor models can range from full functional models that mimic the internal architecture of the processor to simple bus functional models that only describe the interaction of the processors with external components but do not mimic the internal architecture [22]. The former is usually too expensive in terms of execution speed and also difficult to construct from scratch for complex processors. The latter approach is more suitable for debugging the hardware portion but not well suited for viewing software execution. An intermediate approach is to use an instruction set simulator (ISS) coupled with a bus functional model (BFM) to model the processor, such as described in [23]. The ISS will be used to simulate software execution while the BFM will mimic the interaction with the external circuitry. Synchronization techniques between the ISS and the BFM are needed to keep the simulation realistic.

Numerical simulations can be performed at the algorithmic level by generating Matlab code. A Matlab program is generated by selecting the "**.M" file representing each of the processing leaf nodes. A combined Matlab program is generated that is a numerically accurate version of the data flow diagrams. With the proper Matlab functions, precision effects on numerical accuracy can be studied. This capability is used to verify algorithm correctness of the data flow graph as drawn.

\section{SYSTEM SYNTHESIS}

By the time the user is ready for synthesis, the tools have been used to capture system requirements, design information and alternatives, and the resources available for system implementation in the form of Models. The constraints developed during the design representation phase, have been applied to the design space to define a manageable set of implementation alternatives. Expected performance has been estimated using the CoSimulation tools, providing further assurance that the system will function to design specifications. The selected design alternatives must now be transformed to software and hardware for system implementation. We refer to this process as the model interpretation/system synthesis phase.

A model interpretation process generates hardware architecture specifications, software modules, process/schedule tables, communications maps, synthesizable hardware specifications, and a runtime Configuration Manger for dynamic adaptation to changing environments. The synthesis process attempts to optimize hardware/software architectures for user-definable cost functions such as weight, power, algorithmic accuracy and flexibility. 


\section{Configuration Manager Synthesis}

At this point, the synthesis procedure can generate the actual runtime artifacts. The state-based behavior of the system was defined in the Behavior Models. From the behavioral models, a compact state table is produced for the Configuration Manager. The table contains next state equations for each operational mode. Interfaces to internal and external events are generated that provide the state transition variables to the state machine. These tables and variable interfaces are created in a form to allow direct execution by the configuration manager. The Configuration Manager core library is compiled along with the compact state table to generate an executable configuration manager. This configuration manager will be executed on the system control processor.

\section{Hardware Synthesis}

For each configurable component (FPGA), a design specification is generated. This design specification includes a hardware design file for each mode. The design for a component for each mode is specified in structural VHDL. The VHDL design incorporates computational components from the design library. The library can contain user-defined VHDL behavioral/structural descriptions and vendor-supplied Intellectual Property (IP) modules. These modules are combined using components from a standard interface runtime library. The interface library is a key component of the Runtime Environment, described later. These interfaces connect computational components on the same chip with simple FIFO's and asynchronous handshaking interfaces. When the communication must occur across chip boundaries, or between software and hardware components, a set of more complex interface components are used. These interface components manage the physical hardware resources (pins and wires), buffer data, and multiplex multiple logical communications across a single set of wires. Where required, data format conversions are supplied.
These VHDL files are then compiled using vendor-supplied/COTS VHDL compilers and part-specific Place-and-Route tools. The result is a set of "bitfiles". One bitfile is generated for each reconfigurable hardware device for each mode. Given the current state of the FPGA market, demand has not yet forced the vendors to provide partially reconfigurable devices and support tools (Xilinx Virtex parts are said to be partially configurable, however the compiler/PPR tools have not shown documentation and support for this mode of operation. Earlier generations, the XC62xx family, were dynamically partially reconfigurable, however support has been dropped for these chips). For these reasons, we treat each FPGA as an atomic part, configurable only with a full device reset. A partially reconfigurable device is simulated by aggregating multiple standard fully reconfigurable FPGA's. The approach proposed here will work for partially reconfigurable devices when the tools become available. In order for this approach to work with standard compilers/PPR tools, the vendor tools must provide methods for floor planning to restrict logical design components (i.e., all components within a single mode) to non-overlapping, regions that coincide with legal chip reconfiguration boundaries.

\section{Software Synthesis}

For the general-purpose RISC/DSP components, a set of software implementations is generated. These implementations provide the information needed by the Runtime Environment to enact the desired computational behavior. The Runtime Environment requires several categories of design files:

- Software Load Modules contain executable modules that are downloaded to the processors in the system. The system can generate a common load module that contains the superset of all executable functions (if memory is sufficient) or it will generate a customized module for each of the processors in the system. 
The customized module is clearly more memoryefficient.

- Real-time schedules contain the list of processes and their priorities. A unique schedule is generated for each processor and for each mode of operation.

- Communication maps describe the information flow between processes. These "streams" can perform communication between two modules on the same processor, or they can transport data across the network, through intermediate processors, and to a remote process anywhere in the system.

Interfaces between software modules and hardware modules, and data sources/sinks are automatically inserted during the synthesis process. These interfaces perform the management of hardware interfaces, converting complex communication protocols into simpler hardware compatible protocols. The interfaces also multiplex multiple logical streams over a single physical port and perform data conversion functions. It is the responsibility of the synthesis process to ensure that adequate bandwidth exists on each port for the data flow through that port.

These design files are processed into a set of object modules and tables for inclusion in the configuration manager and for direct download into the array of parallel processors.

The result of the synthesis and post processing is a complete executable system, ready for deployment. The deployment is performed in concert with the Runtime Environment.

\section{RUNTIME ENVIRONMENT FOR DYNAMIC RECONFIGURATION}

The runtime environment must support implementation platforms with the following attributes:

- Heterogeneity Optimizing the architecture for performance, size, and power requires that the most appropriate implementation techniques be used. Implementations will require software (implemented on RISC and DSP processors), configurable hardware on FPGAs, and a mix of ASIC components.

- Low Overhead/High Performance The runtime environment must minimize overhead, since overhead results in extra hardware requirements.

- Hard Real-Time The target systems have significant real-time constraints.

- Reconfiguration The execution environment must allow hardware and software resources to be reallocated dynamically. During reconfiguration, the application data must remain consistent and real-time constraints must be satisfied.

These issues must be addressed at multiple levels. At the lowest level, the hardware must be capable of reconfiguration. Software-programmable components, such as DSP's and RISC processors, have excellent inherent hardware support for reconfiguration, since software has the ability to change system function by changing memory contents. Internal CPU hardware structures are designed to restrict dangerous conditions that could damage hardware. FPGA's, on the other hand, are an unrestricted collection of gates, switches, and connectors. The safeguards built into CPU's do not exist and must be enforced manually. This protection must be provided by a cooperation of the design process and the runtime infrastructure.

At a slightly higher level, the internal state of software must be managed under changing tasking. Modern operating systems have evolved to support the flexible implementation of multiple tasks, with dynamic addition and removal of tasks on a single processor in the form of time-sharing and/or multitasking, and Real-time kernels allow time critical tasks to be dynamically scheduled on a single processor. These kernels typically do not address the consistency of dynamic reconfiguration for distributed networks of tasks. Finally the issues of application-specific requirements must be addressed, to allow the peculiar 
requirements of specific numerical performance and timing to be achieved in an implementation. Potential solutions to these issues with consistency are addressed in the next section.

\section{Hardware/System Consistency During Reconfiguration}

The runtime system must avoid operational defects during a reconfiguration event. Lack of hardware consistency can have many negative effects, from temporary loss of performance in an operational mode to hardware damage and total, permanent system malfunction. Typically, these deal with specific issues involving interfaces between hardware processes and/or devices. Some of these defects are illustrated in Figure 5.

Port contention occurs when bi-directional ports are improperly initialized, a reconfiguration event is not properly sequenced/synchronized, or if an improper/inconsistent design is implemented. In this case, two connected drivers are enabled. If resistance is sufficiently low, permanent physical damage can occur to the circuits.

Token loss or duplication results from incorrect initialization or a loss of communication integrity. Tokens represent the status of empty or full slots in a communication interface. An extra token on the sender side can cause too much data to be sent, resulting in a FIFO overrun. A lost token can effectively block a communication port, resulting in a system deadlock.

Device state maintenance refers to the control of a complex external hardware device, such as an attached processor or storage device. In controlling an external device, the controlling computational component must maintain an accurate representation of the device's state. If a reconfiguration occurs during a state transition within the device, or if the reconfiguration modifies the computational component's representation of the device, there can be a state mismatch. This can result in improper commands being sent to the device, or in a deadlock where both components are waiting on each other for triggering events.

These three examples show some of the potential hazards that can occur when the hardware device is improperly reconfigured. Runtime reconfiguration support must not permit any of these conditions to occur.

\section{Software/OS Consistency}

Software issues can present a larger challenge to dynamic system reconfiguration. While the hardware built into standard microprocessor devices protects against low-level hardware conflicts, there are many more details that must be managed. Figure 6 below summarizes some of the potential problems from an improper reconfiguration.

\section{Hardware Consistency After Reconfig}

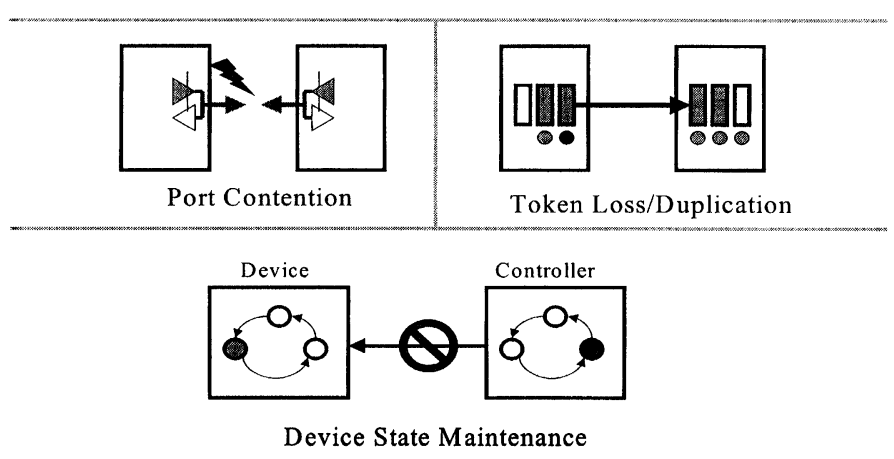

FIGURE 5 Hardware reconfiguration problems: maintaining consistency. 


\section{Software/OS Consistency on Reconfig}

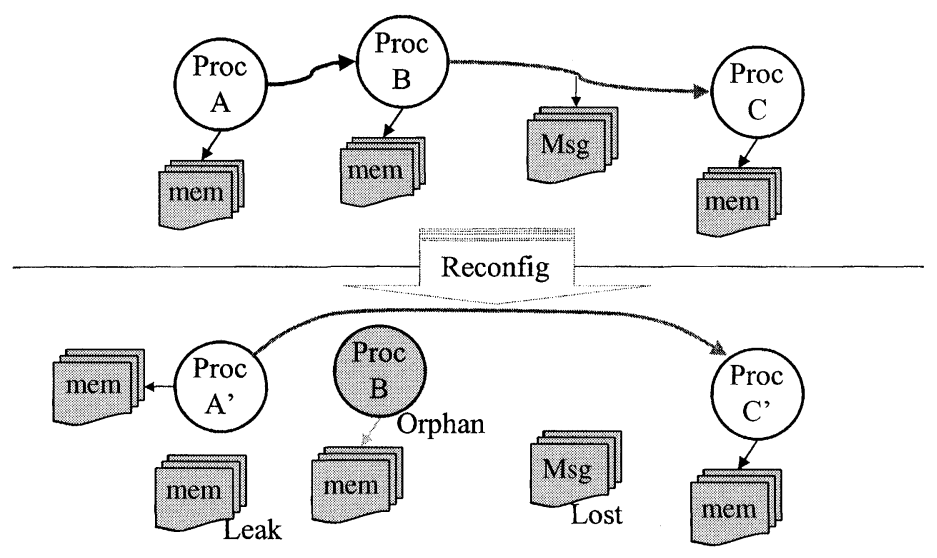

FIGURE 6 Software/OS reconfiguration problems: maintaining consistency.

The example shows an initial configuration of 3 processes (A, B and C) in the normal operational state. A reconfiguration occurs, changing to a new configuration. The new configuration replaces these process $\mathrm{A}$ with $\mathrm{A}^{\prime}, \mathrm{C}$ with $\mathrm{C}^{\prime}$ and removes Process $\mathrm{B}$ altogether. The bottom half of the figure shows the new configuration, along with the potential errors.

Memory leaks will adversely affect long-term reliability. Task structure mismanagement results in extra tasks executed by the kernel, with a loss in performance. Messages in transit can be delivered when the receiving process no longer exists, resulting in mis-matched messages and communication errors.

\section{Application-level Consistency}

At a higher level, the application's requirements and implementation details impose restrictions in the reconfiguration process. Typically, these attributes are highly application-specific. Two examples of consistency requirements are displayed in Figure 7 below.

1. An external system may require signal output continuity and/or continuous first derivative properties. In the example, which swaps filters online, the new filter is operating out of sync with the original filter. A rapid switchover will create a discontinuity in both the signal and its first derivative. In a closed-loop system this might lead to strong transients in the controlled variable.

2. The system can fail to maintain real-time constraints during reconfiguration. If the reconfiguration cannot be completed in sufficient time, deadlines will be sacrificed. In addition, the timebase can be shifted, resulting in a skew in system output period.

\section{Runtime Reconfiguration Strategies}

It is clear that reconfiguration support must be built into the design approach, from the lowest levels of the execution environment, to the highlevel design/requirements capture tools. The extent of support is defined by the requirements of the target systems. The driving factors include how fast the system must reconfigure, whether intermediate states must be preserved (Application Signal Continuity), and if timing must be preserved. We now examine the potential reconfiguration strategies and their impact on system capabilities. 


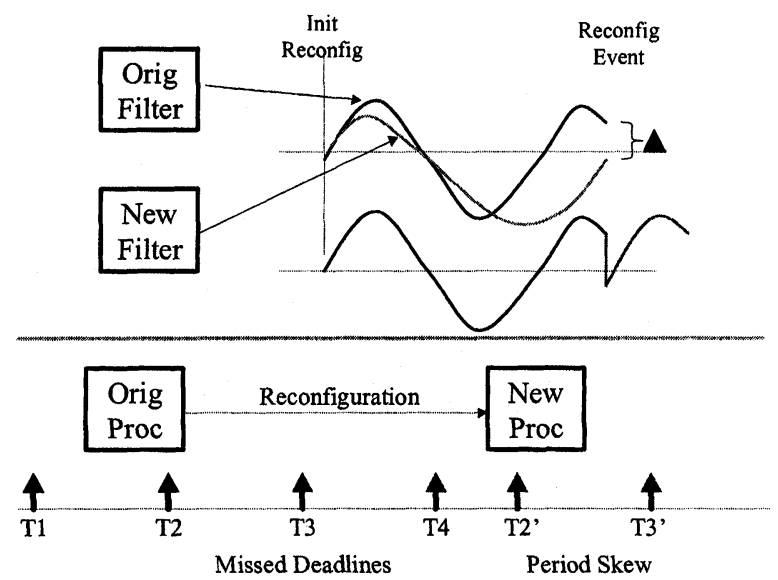

FIGURE 7 Maintaining application consistency through reconfiguration.

\section{Reboot Strategy}

The simplest reconfiguration strategy is termed the "Reboot" approach. It involves the orderly shutdown of tasks, bringing the system to a known, clean state. From this state, a new processing structure is constructed (Fig. 8). The implementation for this approach is simple, requiring the minimum amount of non-standard support from the execution environment and there is no need for additional processing capability for overlapping modes.

The drawbacks of this approach are severe. The system is offline during the reconfiguration time. No events can be handled, so a system under control is open-loop during that time. There is no provision for preservation of state. This can lead to long recovery times when the new configuration is started. Both of these factors lead to system application transients, both in timing and signal continuity. This approach is not suited for the majority of embedded, closed-loop systems.

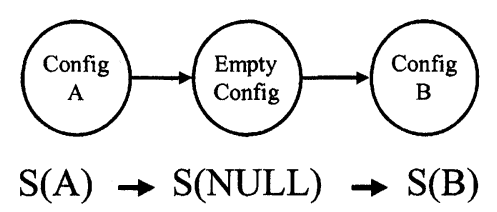

FIGURE 8 Reconfiguration strategies - "Reboot" approach.

\section{State Transition Approach}

The second approach allows the insertion of transitory states between the major system operating modes (Fig. 9). These states allow the system to take smaller steps between operational modes to approximate a continuous-time transition, resulting in smaller transients. The intermediate configurations inherit state from their predecessors. The intermediate algorithms must be designed to gradually shift system behavior. While not continuous, the steps can be made arbitrarily small.

This approach has several positive aspects. The state preservation allows transients to be minimized. The magnitude of the steps can be chosen by the designer to minimize key application behaviors. Few spare resources are needed, since the system is operating in only one mode at a time. The flexibility is limited only by the designers and by the time available for the transition.

There are several difficulties in this approach: The execution infrastructure must support the

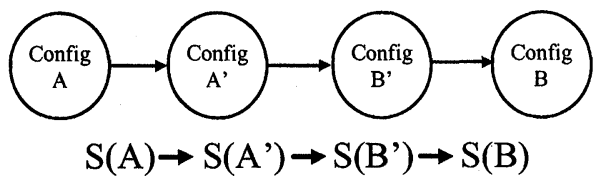

FIGURE 9 Reconfiguration strategies - state transition approach. 
rapid transition of processes and transition of the states of the changing processes. The states must be mapped to the structures required by the next step, and installed with the new processing structure. The computation of the mapping may be complex.

The design of intermediate states can be complex, depending on the application. These transitory states depend both on the initial state and the final state, the algorithm characteristics, and the timing requirements. For smooth application transitions, many intermediate states may be required, leading to long transition times. (It should be noted that the application system is still under control during transition, but probably not the optimal algorithm.)

\section{Parallel State Transition Approach}

An extension of the State Transition approach allows the system to execute several modes in parallel. This has the same benefits as the state transition approach with the added benefit of being able to execute algorithms prior to use, in an offline mode. The state of the offline process can be allowed to stabilize prior to impacting upon system performance. When transients have disappeared, the system can be transitioned to the new state (Fig. 10).

This approach has several benefits. The application-level transients can be minimized by proper design. The downtime is minimal, as is the operation of the system in a less-than-optimal configuration. Multiple states can be preserved, not forcing all information to be encoded in one

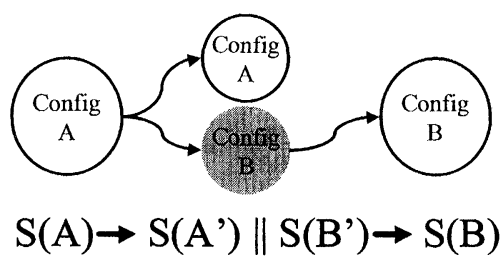

FIGURE 10 Reconfiguration strategies - "Parallel execution" approach. format. This minimizes the impact of the design of one mode on another, thus simplifying design.

There are also several drawbacks. The underlying runtime environment must support mechanisms for rapid stepping between processes, the ability to execute multiple threads simultaneously, and the combination of attributes from the parallel executing processes. System design is complicated by the need to design parallel structures. (In some cases, the parallel approach allows design separability, simplifying matters.) The neces-sary computational resources are increased, due to the need to execute multiple parallel processes.

\section{Execution Environment Design}

The previous sections assembled a set of requirements for the execution environment. They also point out some of the design complexities. Working alone, the execution environment cannot solve these problems. The overall system design approach must coordinate from the top-level algorithm designers/system requirement and resource specifications down to the hardware/software implementations. The top-level design issues have been discussed in terms of a domain-specific modeling environment, where the environment is tuned to reconfigurable system design. The Execution Environment forms the infrastructure onto which these designs are projected.

The Execution Environment must be designed with an interface suitable for synthesis from a MIC-Generator approach. The concepts, properties and interfaces of the runtime environment must be compatible with the design representation and synthesis approach. Capabilities and interfaces should be tuned to simplify the generator. This requirement demands a simple, uniform interface with a well-defined, consistent set of semantics that apply throughout the system. Since the system includes software, hardware, and interactions between parallel modules, a common structure must map to a wide range of components. 
The execution environment concepts have been driven by results from using tools developed over the past several years. These tools are currently used to construct large-scale, parallel, real-time signal processing systems. The runtime environment enabled development of CADDMAS systems, which are used by the USAF for turbine engine testing and NASA for SSME monitoring and analysis $[9,19]$.

The semantics of the execution environment implement a large-grain-dataflow architecture. The Worker Function captures the tasks that are performed by the system. Communication nodes capture the transfer of data between workers. Computations can be described as a bipartite graph, where workers connect to Comm nodes, and Comm nodes connect to workers. At this level, there are no implied semantics of the workers. The execution properties of workers (Data tokens produced/consumed per execution, timing of execution, etc.) are maintained at a higher level. The semantics of the Comm units are asynchronous queues.

When the generic large-grain dataflow graphs are implemented, they must be mapped down to a physical implementation. The implementation takes the form of either software or hardware. Software workers execute on a DSP or CPU, which we term Processes. Hardware workers are either implemented in reconfigurable hardware (FPGA's), or ASIC implementations, or combinations of both. Software Processes and Hardware Processors are logically equivalent, representing functions on data. Processes/Processors are connected via logical Comm that must buffer, communicate, and match data formats. In software implementations, the Comm object is logical Stream. implemented by the Kernel as a software queue in memory. In hardware, the Comm object is implemented with registers and/or FIFO, or simply wires (Fig. 11).

The execution environment spans software and reconfigurable hardware. The software environment consists of a simple, portable real-time kernel with a run-time-configurable process schedule, communication schedule, and memory management [19]. Communications interfaces are supported within the kernel, making cross-processor connections invisible. Memory management is integrated with the scheduler and communication subsystems, enabling (but not solving) the problems associated with dynamic reconfiguration. The kernel allows dynamic editing of the process table, and of the communications maps. The proper sequencing of these operations, including task execution phases, is necessary for the avoidance of reconfiguration problems. The current approach supports the "Reboot" approach directly, and will support the more advanced reconfiguration approaches with cooperation of the application tasks.

The hardware execution environment supports the same operational semantics. The implementation, however, is much different. The Virtual Hardware Kernel exists as a concept used in the system synthesis. The MIC Generator synthesizes a set of VHDL structural codes, one for each configurable device multiplied by the number of operational modes. Hardware Processors are directly synthesized using predefined components. Communications elements are selected from a library of interface types, based on the requirements of the workers on either end, the required performance, and the available resources. The communication infrastructure works in cooperation with the software communications, performing the signal buffering, and the necessary off-chip interfaces and data converters. The interface components are drawn from a library of modules. The modules implement a limited set of standardized communications protocols to transfer data between modules, and present data in the format required by the destination processor. As the system is used for more applications, the set of interface types will grow in capability.

Inherent in these interface components must be the capability to reconfigure. This involves strict synchronization mechanisms, methods for saving and restoring states, and facilities to allow function and structure modification. Global system 


\section{Common Execution Semantics}

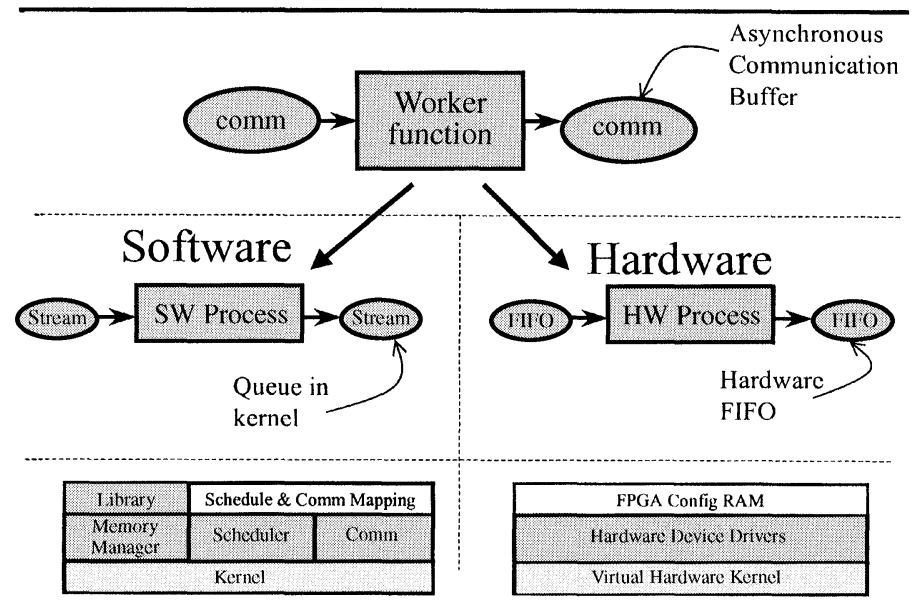

FIGURE 11 Runtime execution environment: common execution semantics.

synchronization is greatly aided by having a common system clock, and facilities for very lowlatency signaling within the system. Our current concepts for reconfiguration require a single interrupt signal to be present at each component participating in a reconfiguration.

In addition, the runtime environment must be designed with an interface suitable for synthesis from a MIC-Generator approach. The properties of the runtime environment must be tuned to simplify the generator. This demands a simple, uniform interface with a well-defined, consistent set of semantics that apply throughout the system.

\section{Reconfiguration Manager}

The reconfigurable hardware interfaces, and the flexible microkernel provide the facilities to implement system reconfiguration, however the problem of control and synchronization is critical. A global view of the system is necessary. The kernel, in isolation, cannot perform reconfiguration.

Synchronization and control of a system during reconfiguration is the responsibility of the Configuration Manager (CM). The CM contains tables capturing the behavioral state machine defined by the designers Behavioral Models. Tied to these state-based descriptions is the information necessary to configure the hardware and software components of the system.

Given this information, the $\mathrm{CM}$ serves as a system observer. The CM monitors relevant signals, as defined in the transitions leading out of the current state. When the logical conditions for a state transition are satisfied, the CM begins the structural transition process.

The first stage of the reconfiguration involves transitioning the system into a known, safe state. All communication interfaces must terminate. Since many of the data ports are bi-directional, the bus direction control token must be returned to the 'safe' state. Computations must be completed and transitioned into the 'safe' state. The safe state may involve using local algorithms to perform the basic required functions to keep the system stable.

After all necessary components are in the safe state, the global interrupt is toggled to initiate the reconfiguration event. At this point, all communications must stop for the short period required for reloading the FPGA's bitfiles and the Software schedules and communication mappings. Since the state of the system was in a known safe state prior to reconfiguration enactment, 
there is little overhead atop the basic information download. The CM will reload the necessary FPGA's using the standard download methods. A sequence of commands is sent to each of the processors to enact the new processing graph and interface components. Once the new programming information is installed, the system interrupt signal is toggled to ensure a globally synchronized start up operation.

\section{APPLICATION EXAMPLE}

The design environment has been used for several applications. Here, we will describe an Automatic Target Recognition application for missiles. The application is highly resource constrained, has hard real-time requirements, and must function in multiple operational modes.

The initial design process involves iteratively constructing models that capture system design information. The ATR application design first specifies system operational requirements in the form of Behavioral Models. Figures 12, 13 show these top-level behavioral models for the missile behavior. From the Initial transition, the system enters into the INIT phase (upper left). The system transitions into the 'Ready' state, where it waits for signals from the operator. The 'Seek Target' signal will start the active system operation in a 'On Platform Target Seek' where the controller locates a target then waits for a 'Launch' command before transitioning to the 'Tracking' state. A 'Launch' signal can also cause the system to transition directly from the 'Ready' to the 'Tracking' state. Figure 13 shows the internal composition of the 'Tracking' state. The system enters the 'Tracking' via the 'LOBL' (Lock-onBefore-Launch) or 'LOAL' (Lock-On-AfterLaunch) transition input. The LOAL input transitions directly into 'Acquire_LongRange' mode, where a many-target acquisition is performed, and a target is selected. The system then enters into the long range tracking, until either the track is lost (proceed to 'Acquire_LongRange'), or proximity sensors signal the system to transitions into the 'Tracking_MidRange' state. This process repeats itself for Mid-Range and ShortRange modes.

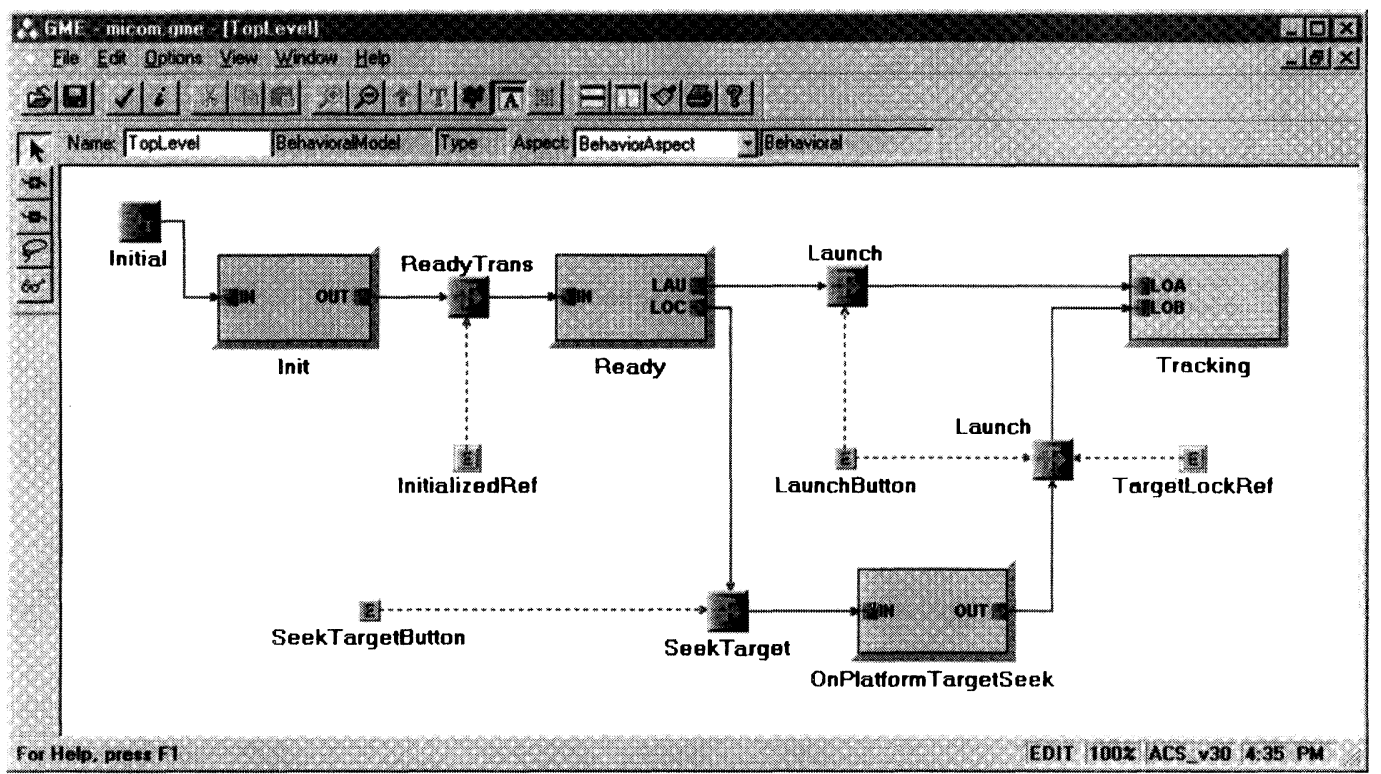

FIGURE 12 Top-level behavioral model. 


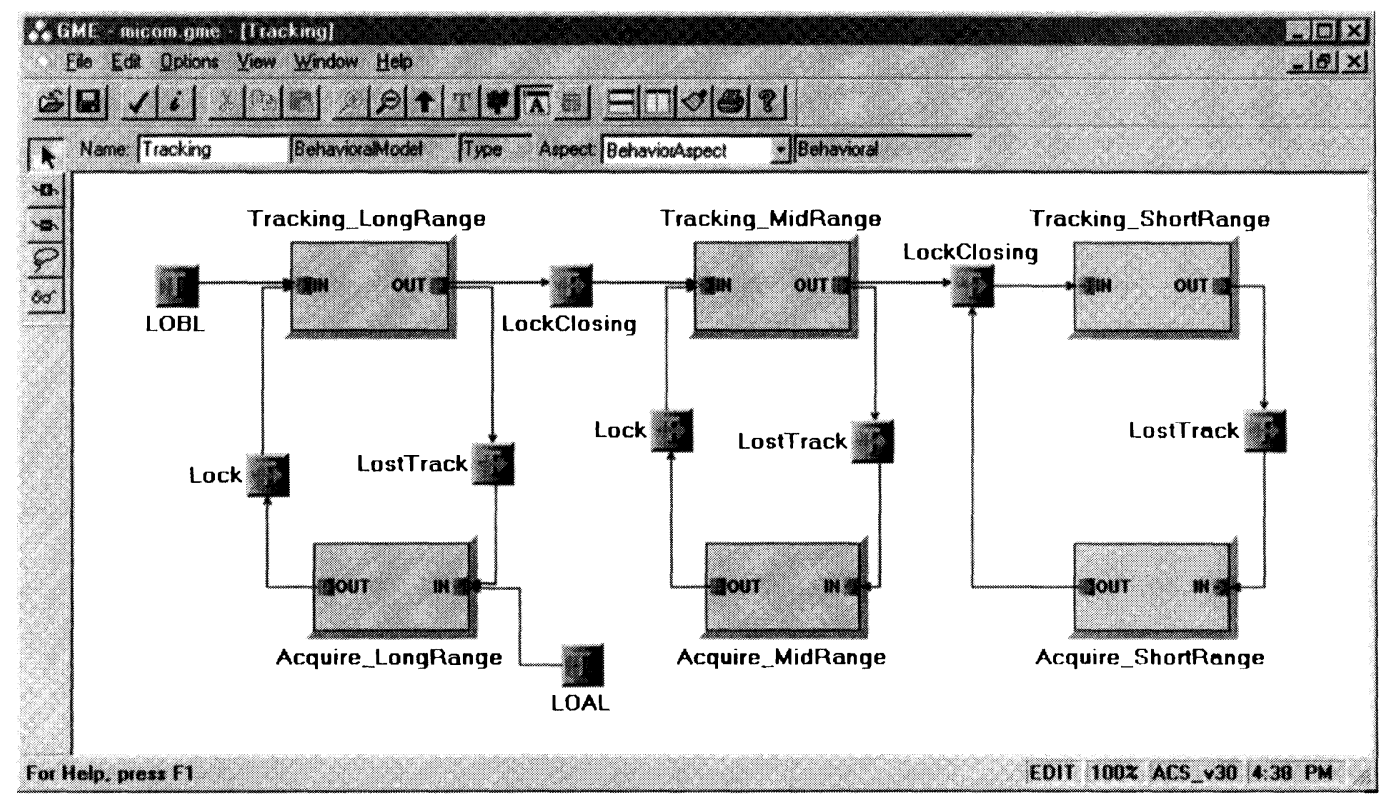

FIGURE 13 ATR behavioral model, tracking drill-down.

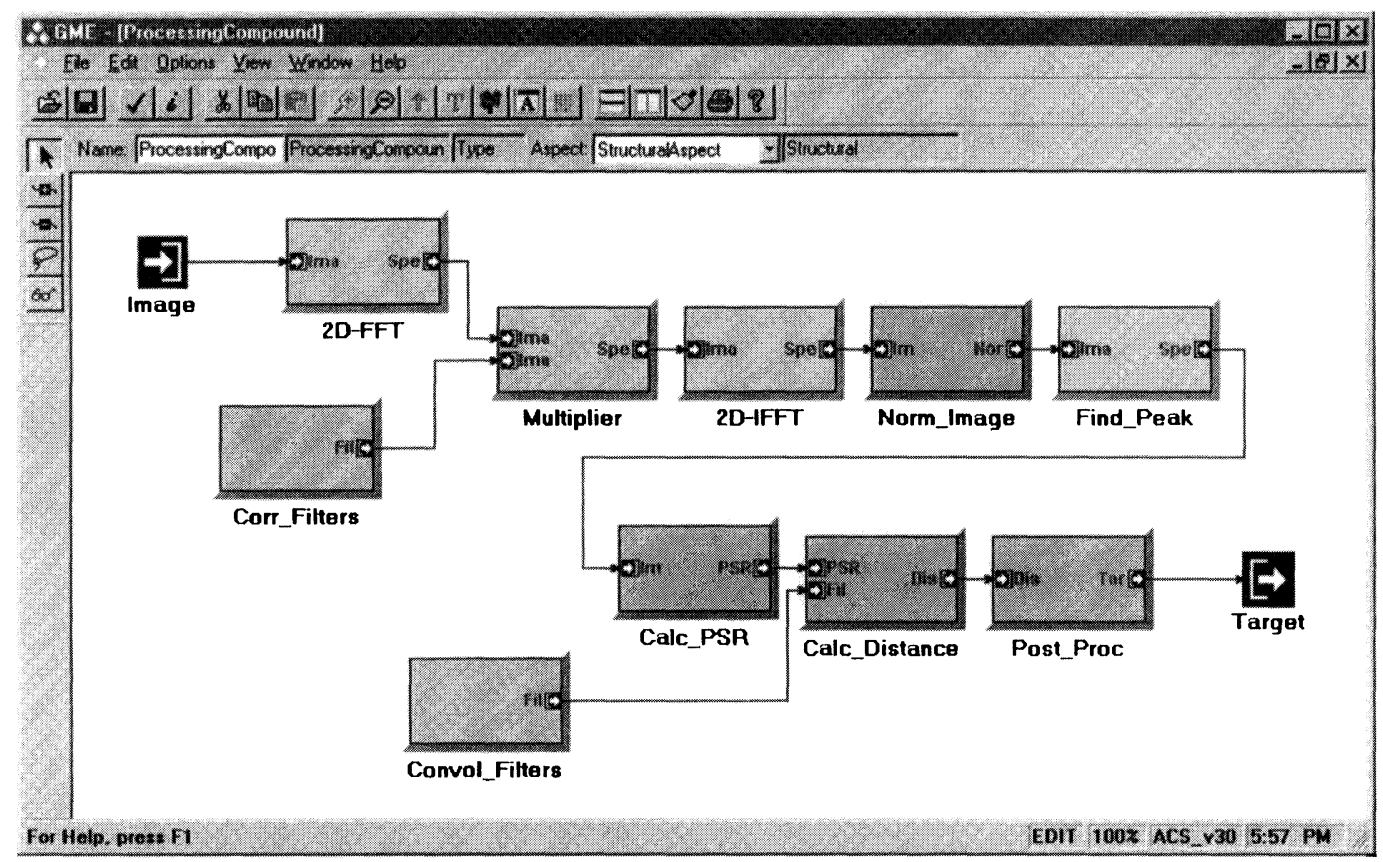

FIGURE 14 Top-level ATR algorithm structural models.

In parallel with the definition of the behavioral requirement, signal-processing engineers can define algorithm structures in the Algorithm Models using a library of components. Hierarchy allows multiple designers to work at different levels in the design space. Figure 14 shows the top-level signal flow for the long-range target acquisition modes. 


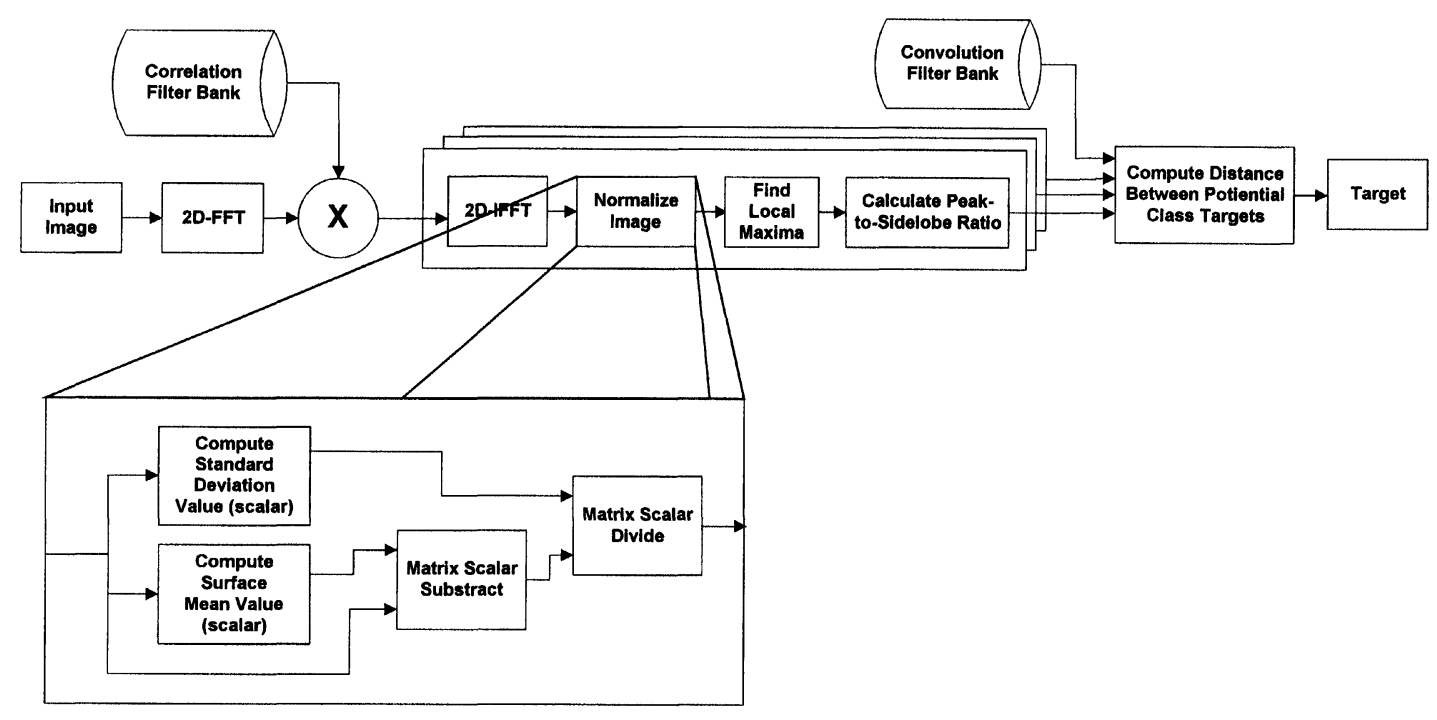

FIGURE 15 ATR algorithm signal flow.

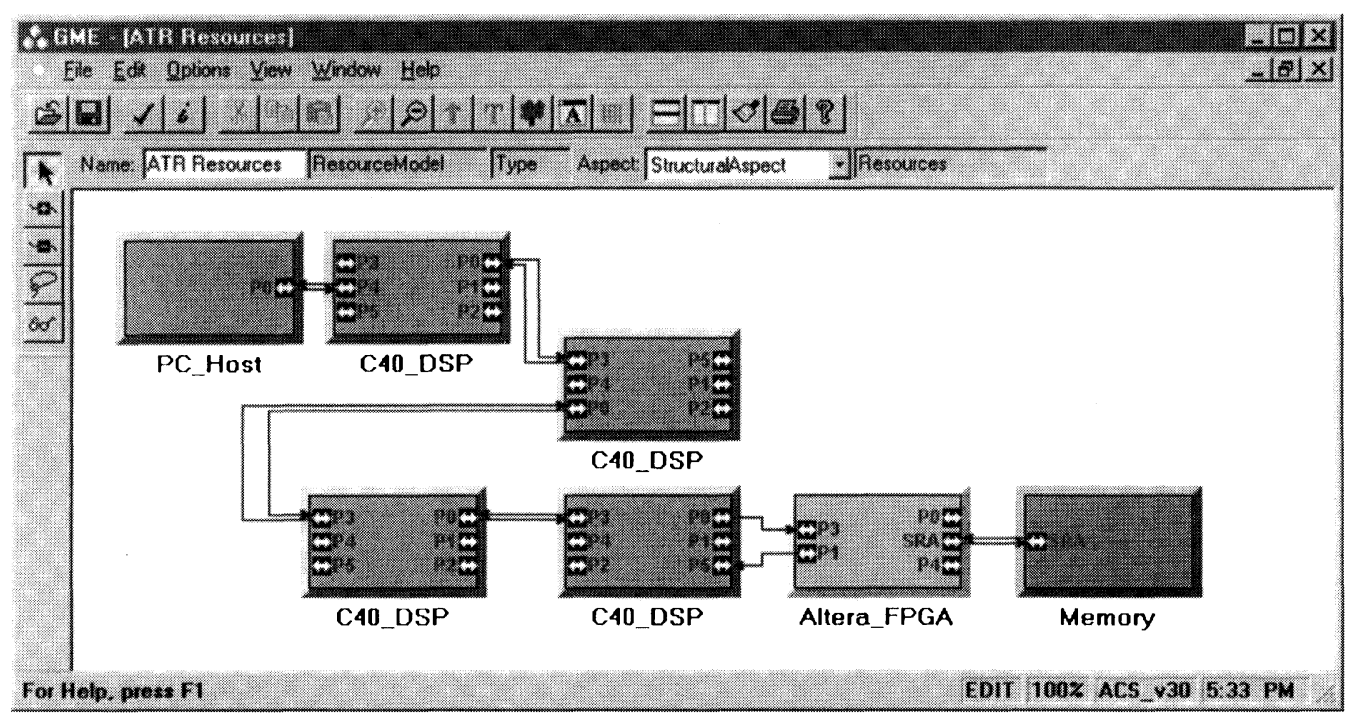

FIGURE 16 ATR hardware resource models.

These algorithms are described in a model hierarchy, using Compounds, Templates/Alternatives, and Primitives. Where possible, libraries of preexisting components are used. When new components are required, signal processing engineers and hardware VHDL designers develop or acquire modules and capture implementation attributes, such as benchmark results, into the design environment's component libraries. In the tracking algorithm, several components were developed for hardware in VHDL and software (C for the TMS320C40). Figure 15 shows the top level Algorithm Models of the ATR application. 


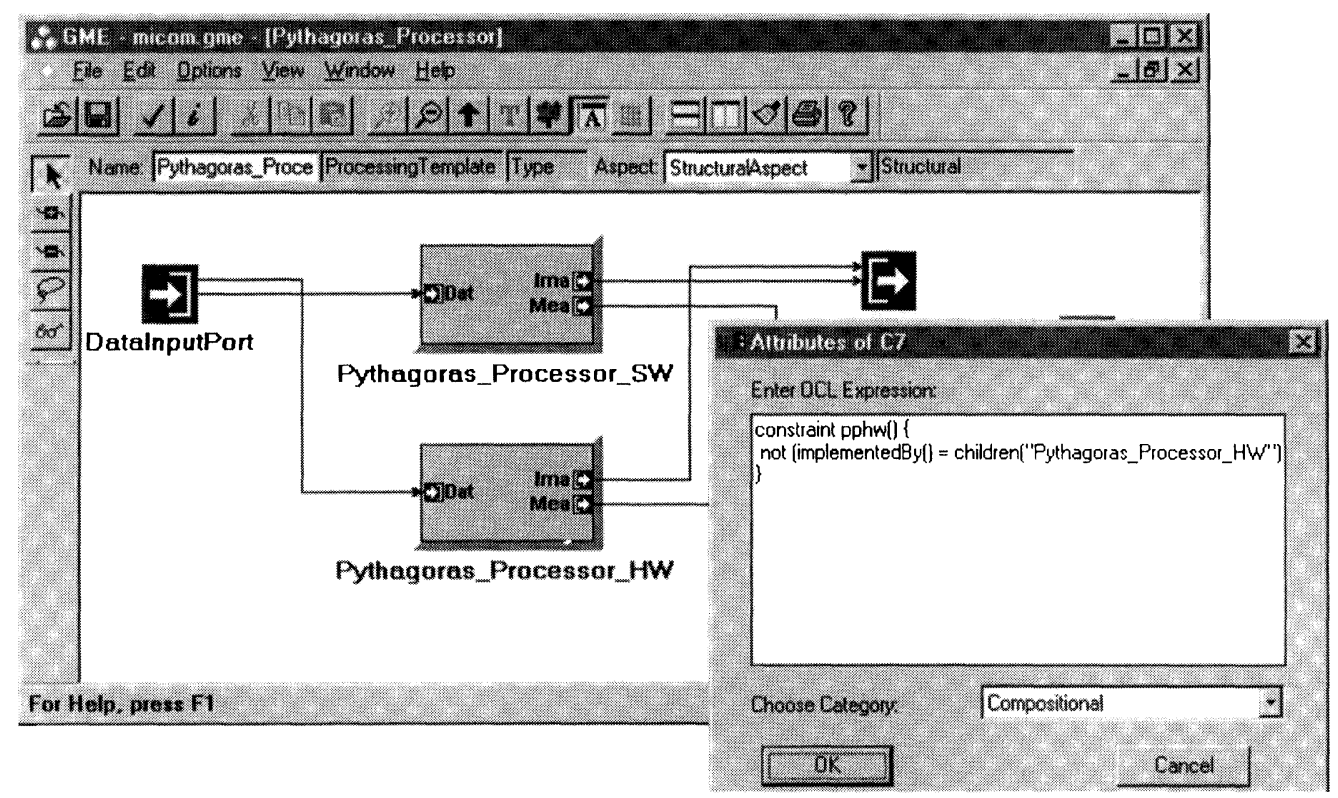

FIGURE 17 ATR constraint specifications.

In parallel with design of Behavioral and Algorithm Models, hardware engineers are capturing the hardware architecture details in the Resource Models. If the system is to be constructed with flexible hardware modules, the specifics of these modules are captured and the final assembly can be left for future specification. Where the boards are hardwired, the complete topology is captured directly. Figure 16 shows the top level of the Resource models. This figure shows the 1 FPGA, its external memory, 4 DSP processors, and 1 RISC processor available for target tracking.

Assigning Algorithm Models to Behavioral Models, and assigning Resources to Behavioral Models and Algorithms assembles the component models. These assignments are specified by 'dragging' one model into another as a Reference. Constraint specifications are developed to express complex relationships. See Figure 17.

The models are analyzed with the symbolic constraint manager to explore the design space. The initial design space in the ATR algorithm is $10^{24}$. The constraints are iteratively applied to reduce the system to approximately $100-1000$ potential configurations. Figure 18 shows the design space size at various stages of the iterative constraint application process.

From the remaining configurations, the designer selects one for implementation. The synthesis process produces an implementable hardware and software design. The VHDL designs are

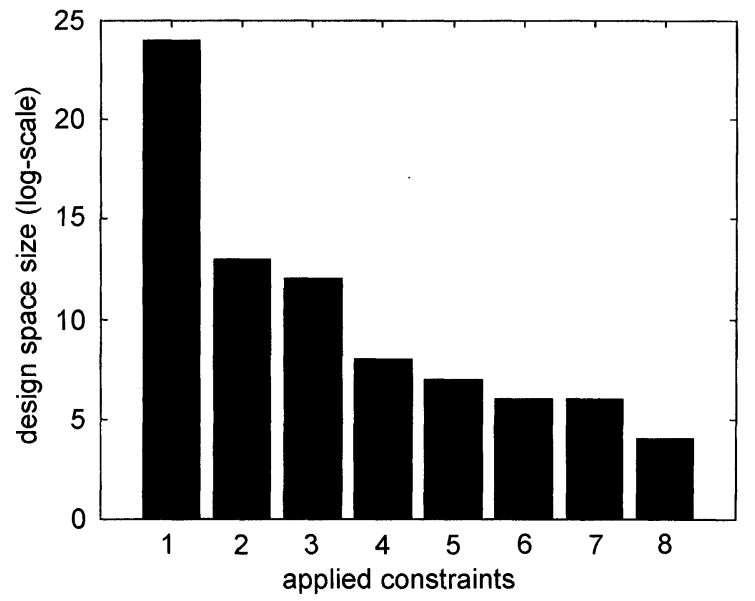

FIGURE 18 ATR iterative constraint application. 


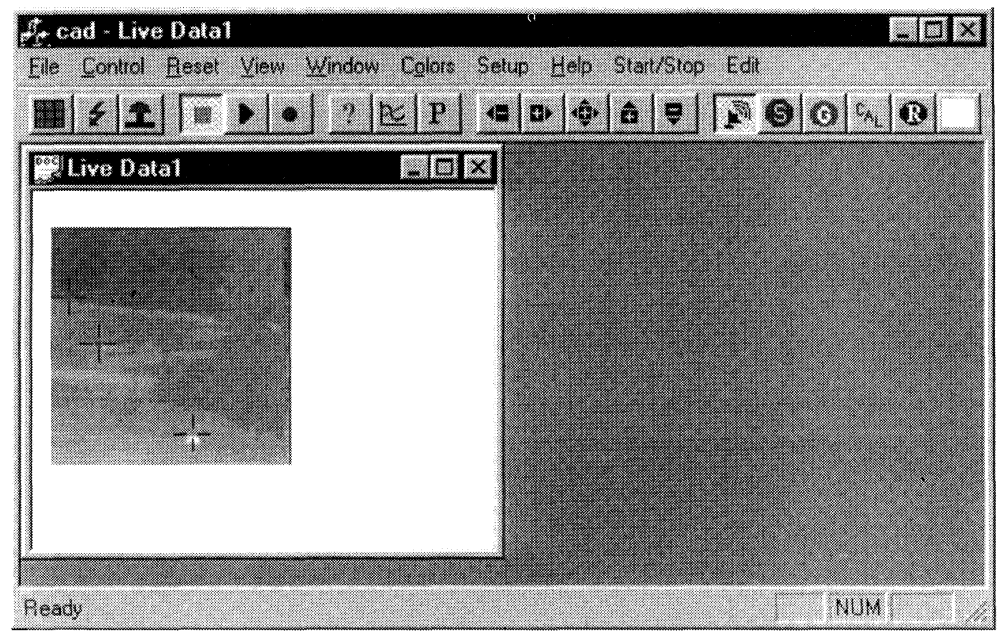

FIGURE 19 ATR testbench display.

compiled using Synopsys for Xilinx and/or MaxPlus2 for Altera. The software structures are processed via the native $\mathrm{C}$ compiler.

Finally, the system is loaded and executed using the configuration manager. Figure 19 shows a testbench configuration with ATR result image and target selection crosshairs displayed on a Windows-based user interface. Intermediate designs can be instrumented with graphical displays to view algorithm internal data structures.

This discussion shows one path through the design process. Typically, the process involves several iterations, to optimize the algorithm performance, resource utilization, and system functional behavior.

\section{CONCLUSIONS}

The system described within this paper represents an ambitious set of goals for a design tool. The design environment is a comprehensive approach to building heterogeneous, real-time, resourcelimited, dynamically adaptive systems. The Model-Integrated approach has been designed to support the many aspects and disciplines of embedded systems design. The flexible representa- tion, analysis and synthesis of systems will reduce design effort and increase system flexibility. The underlying Runtime Environment, through the abstraction of hardware and software details, presents a uniform architecture for system synthesis and application implementation.

This research adds to the state of the art by defining and implementing a cohesive design environment, where construction of dynamically adaptive systems with structural reconfiguration is a primary concern. The combination of behavior, algorithm, and resources provide the breadth to represent the design of a flexible reconfigurable system. The ability to define a broad design space permits the synthesis tools to optimize target systems. The extensive set of constraints and model references give the designer control over the synthesis process and allow user-guided pruning of large design spaces. The design space exploration tools use scalable OBDD's to permit management of large design spaces, allowing rapid iteration over a wide design space. The integration of performance simulation allows users to receive feedback directly, in the native modelbased design concepts. Finally, a unified hardware/software runtime environment allows the hardware and software to be treated as conceptual 
equivalents. Since the abstraction layers are handled at design-time, the runtime efficiency impact is minimized.

There are some limitations in the presented approach. A dataflow model was chosen for representation and execution in the runtime environment. While many data-intensive applications fit this paradigm, many control-oriented applications cannot be modeled or executed efficiently on a dataflow architecture. The OBDD approach to design space exploration typically scales gracefully for large problems, however they are sensitive to variable ordering. Some orderings result in an exponential explosion in number of OBDD nodes. While the end-result of the constraint based pruning is independent of the order of constraint application, the sizes and execution times of the intermediate steps is sensitive to the order in which the constraints are applied. Also, the current runtime environment supports partial reconfiguration only in software and simulates partial hardware reconfiguration with multiple FPGA's. Partial hardware reconfiguration in this design environment awaits devices and vendor tools that fully support these features.

The prototype tool set has been applied to several small-to-medium-sized design projects with significant success. While metrics have not yet been collected, experience indicates high designer efficiency. The tools are still researchquality and several key components are in the process of design and implementation.

The design approach leads to flexible solutions. The implementation architecture is decoupled from the algorithm. Hardware is modeled as a set of generalized resources. These two factors combine to support device technology evolution, with changes required only to the resource models.

The high-level approach will produce greater design efficiencies and code/component reuse. Given an extensive set of component libraries, complex systems can be assembled rapidly. The component libraries can be extended and specialized to very high-level functions by the construction of hierarchical models. The availability of design alternatives within these hierarchical Models will allow the efficiency of these high-level functions to be maintained near the level of a hand-coded implementation.

There are still many major research challenges to be addressed before achieving a fully functional, robust design tool. These issues are:

1. Optimization The current approach involves defining a very large design space and using constraint methods to extract a set of potential design solutions. The process relies on the engineer to manipulate a complex, interrelated constraint network. This process should be assisted by the design environment. Simple tools are planned that show a sensitivity analysis of design space vs. user-defined constraints. This will help to guide the designer to the appropriate constraints that maximize system performance. Taking this a step further, optimization procedures can be implemented to automate the manipulation of system parameters and constraints. In such a non-linear, discretized space, no guarantee of optimization convergence is possible.

2. Methods for assessing the transient upsets that can occur during a structural reconfiguration are needed. These transient assessment tools are needed for predicting both numerical results and for the real-time behavior during reconfiguration.

3. Procedures for rapidly incorporating vendor IP into libraries must be available to ensure that up-to-date components are available for the design. This also contributes to the ease of updating the technologies in the target platform.

4. Significant effort is required to transition the tools from a research prototype to a commercial quality, accepted design methodology and design environment.

\section{Acknowledgement}

This work has been supported by DARPA/ITO under project DABT63-97-C-0020. 


\section{References}

[1] Villasenor, J. and Mangione-Smith, W., "Configurable Computing", Scientific American, June, 1997.

[2] Arnold, J., Buell, D. and Davis, E., "Splash 2", Proceedings of the 4th Annual ACM Symposium on Parallel Algorithms and Architectures, June, 1992.

[3] David R. Martinez, "Real-time Embedded Signal Processing", IEEE Signal Processing Magazine, September, 1998.

[4] Davis, J., Goel, M., Hylands, C., Kienhuis, B., Lee, E., Liu, J., Liu, X., Muliadi, L., Neuendorffer, S., Reekie, J., Smyth, N., Tsay, J. and Xiong, Y., "Overview of the Ptolemy Project", ERL Technical Report UCB/ERL No. $M 99 / 37$, Dept. EECS, University of California, Berkeley, CA 947 20, July, 1999.

[5] Bondalapati, K., Diniz, P., Duncan, P., Granacki, J., Hall, M., Jain, R. and Zeigler, H. (1999). "DEFACTO: A Design Environment for Adaptive Computing Technology", In: Proceedings of the 6th Reconfigurable Architectures Workshop (RAW'99), Springer-Verlag.

[6] Banerjee, P., Shenoy, N., Choudhary, A., Hauck, S., Bachmann, C., Chang, M., Haldar, M., Joisha, P., Jones, A., Kanhare, A., Nayak, A., Periyacheri, S. and Walkden, M., "MATCH: A MATLAB Compiler for Configurable Computing", Technical Report, Center for Parallel and Distributed Computing, Northwestern University, Aug. 1999, CPDC-TR-9908-013. (Also Submitted to the IEEE Computer Magazine, 1999).

[7] Writhlin, M. and Hutchings, B. (1995). "DISC: The dynamic instruction set computer", Field Programmable Gate Arrays (FPGAs) for Fast Board Development and Reconfigurable Computing, John Schewel, Ed., Proc. SPIE, 2607, 92-103.

[8] Bouldin, D., "CHAMPION: A Software Design Environment for Adaptive Computing Systems", http://microsys6.engr.utk.edu/ bouldin/darpa/

[9] Bapty, T., Ledeczi, A., Davis, J., Abbott, B., Hayes, T. and Tibbals, T. (1996). "Turbine Engine Diagnostics Using a Parallel Signal Processor", Joint Technology Showcase on Integrated Monitoring, Diagnostics, and Failure Prevention, Mobile, AL.

[10] Karsai, G., Sztipanovits, J., Padalkar, S. and DeCaria, F., "Model-embedded On-line Problem Solving Environment for Chemical Engineering", Proceedings of the International Conference on Engineering of Complex Computer Systems, Ft. Lauderdale, Florida, Nov. 6-10, 1995.

[11] Long, E., Misra, A. and Sztipanovits, J., "Saturn Site Production Flow (SSPF): Accomplishments and Challenges", Proceedings of the Engineering of Computer Based Systems, Maale Hachamisha, Israel, AL, March, 1998.

[12] Davis, J., Scott, J., Sztipanovits, J., Karsai, G. and Martinez, M., "Integrated Analysis Environment for High Impact Systems," Proceedings of the Engineering of Computer Based Systems, Jerusalem, Israel, April, 1998.

[13] Bapty, T. and Sztipanovits, J., "Model-Based Engineering of Large-Scale Real-Time Systems", Proceedings of the Engineering of Computer Based Systems (ECBS) Conference, Montery, CA, March, 1997.

[14] Carnes, J. R. and Misra, A., "Model-Integrated Toolset for Fault Detection, Isolation and Recovery (FDIR)", Proceedings of International Conference and Workshop on Engineering of Computer Based Systems, Friedrichshafen, Germany, AL, March 11-15, 1996.
[15] Harel, D. (1987). "StateCharts: A Visual Formalism for Complex Systems", Science of Computer Programming, 8, 231-278.

[16] Bryant, R. E., "Symbolic Boolean Manipulation with Ordered Binary Decision Diagrams", Technical Report $C M U-C S-92-160$, School of Computer Science, Carnegie Mellon University, June, 1992.

[17] Bryant, R. E. (1986). "Graph-based Algorithms for Boolean Function Manipulation", IEEE Transactions on Computers, C35(8).

[18] Kumar, S. and Rose, F., "Integrated Simulation of Performance Models and Behavioral Models", Proceedings of the Fall 1996 VIUF, pp. 185-194, Durham, NC, October, 1996.

[19] Bapty, T. and Abbott, B., "Portable Kernel for High-level Synthesis of Complex DSP-Systems", Proceedings of the the International Conference on Signal Processing Applications and Technology, Boston, MA, May, 1995.

[20] Sandeep Neema (1999). "Constraint based System Synthesis", Technical Report, Department of Electrical and Computer Engineering, Vanderbilt University.

[21] Hein, C. and Nasoff, D., "VHDL-based Performance Modeling and Virtual Prototyping", Proceedings of the 2nd Annual RASSP Conference, Arlington, VA, July, 1995.

[22] James Rowson, "Hardware/Software cosimulation", Proceedings of the 31st Design Automation Conference, pp. 439-440, San Diego, CA, June, 1994.

[23] Russel Klein, "Miami: A Hardware-Software cosimulation Environment", Proceedings of the 7th IEEE International Workshop on Rapid Systems Prototyping, June, 1996.

\section{Authors' Biographies}

Ted Bapty is currently working in on tools for Adaptive Computing for dynamically reconfigurable embedded computing applications, on ModelBased Tools for engineering system design, and on tools and systems for parallel, embedded systems. $\mathrm{He}$ is a member of IEEE, the IEEE Computer Society, and serves the Engineering of Computer Based Systems Technical Committee. He is the Director of the Institute for Software Integrated Systems, Vanderbilt University, He can be reached at: bapty@isis.vanderbilt.edu

Sandeep Neema's research interests include Reconfigurable Computing, Symbolic Constraint Processing and Real-time Embedded Systems Programming. He is a research assistant at the Institute for Software Integrated Systems, Vanderbilt University. Nashville, USA, working on his doctoral research. He can be reached at: neemask@isis.vanderbilt.edu

Jason Scott's research interests include reconfigurable systems, and verification of real-time 
systems using symbolic model checking. Jason Scott is a research assistant currently working on his doctoral research at the Institute for Software Integrated Systems, Vanderbilt University, Nashville, TN. He can be reached at: jscott@ isis.vanderbilt.edu

Janos Sztipanovits works in the area of ModelIntegrated Computing for design and evolution of complex computer-based systems. He has chaired several conferences and workshops, including the 1999 IEEE Engineering of Computer Based Systems Conference. He is author of over 130 technical publications. Dr. Sctipanovits is currently a Program Manager at DARPA. He can be reached at: jsztipanovits@darpa.mil

Sameh Asaad works in the area of simulation of hardware-software systems. He is currently a researcher at IBM T. J. Watson Research Facility and a graduate student at Vanderbilt University. 

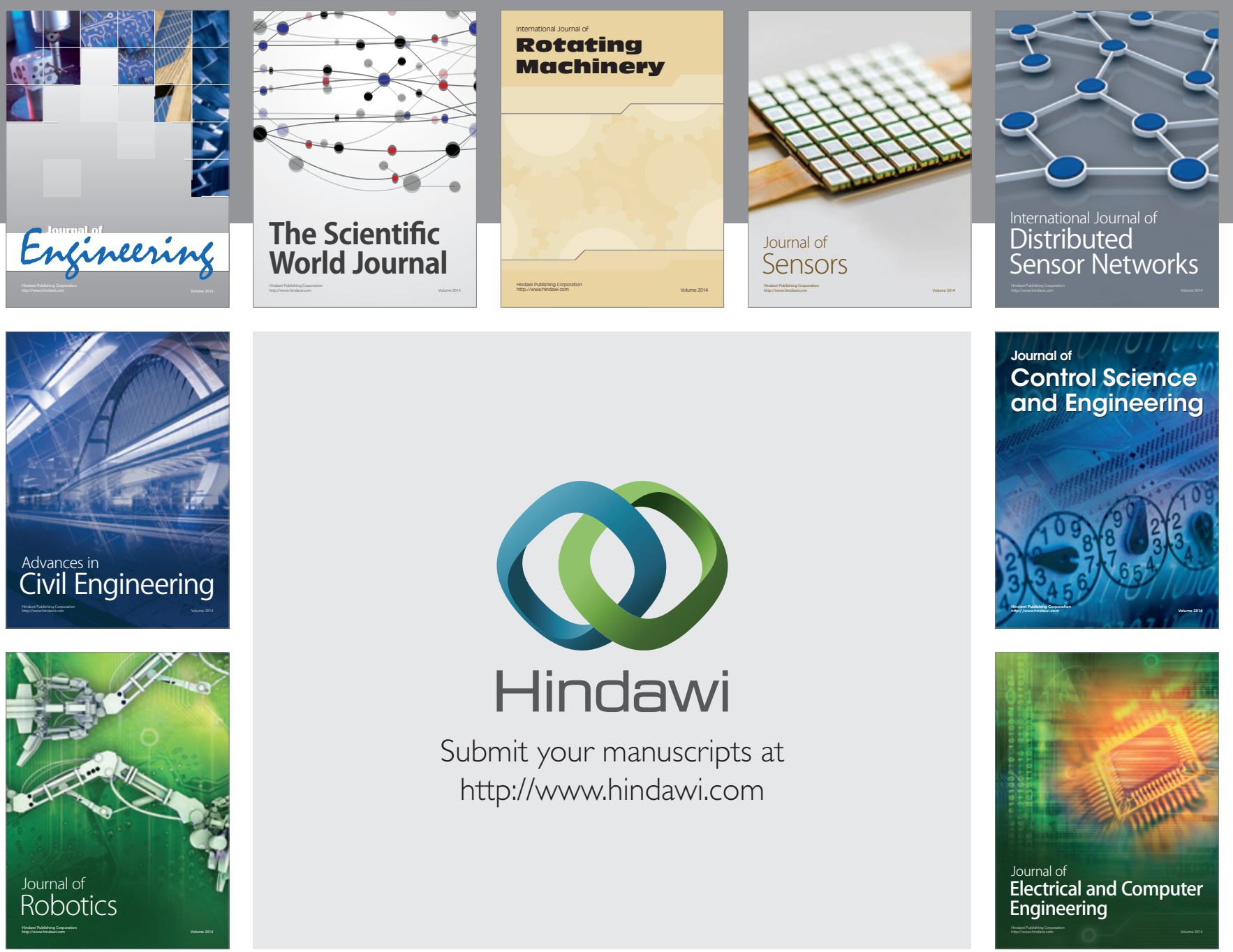

Submit your manuscripts at

http://www.hindawi.com
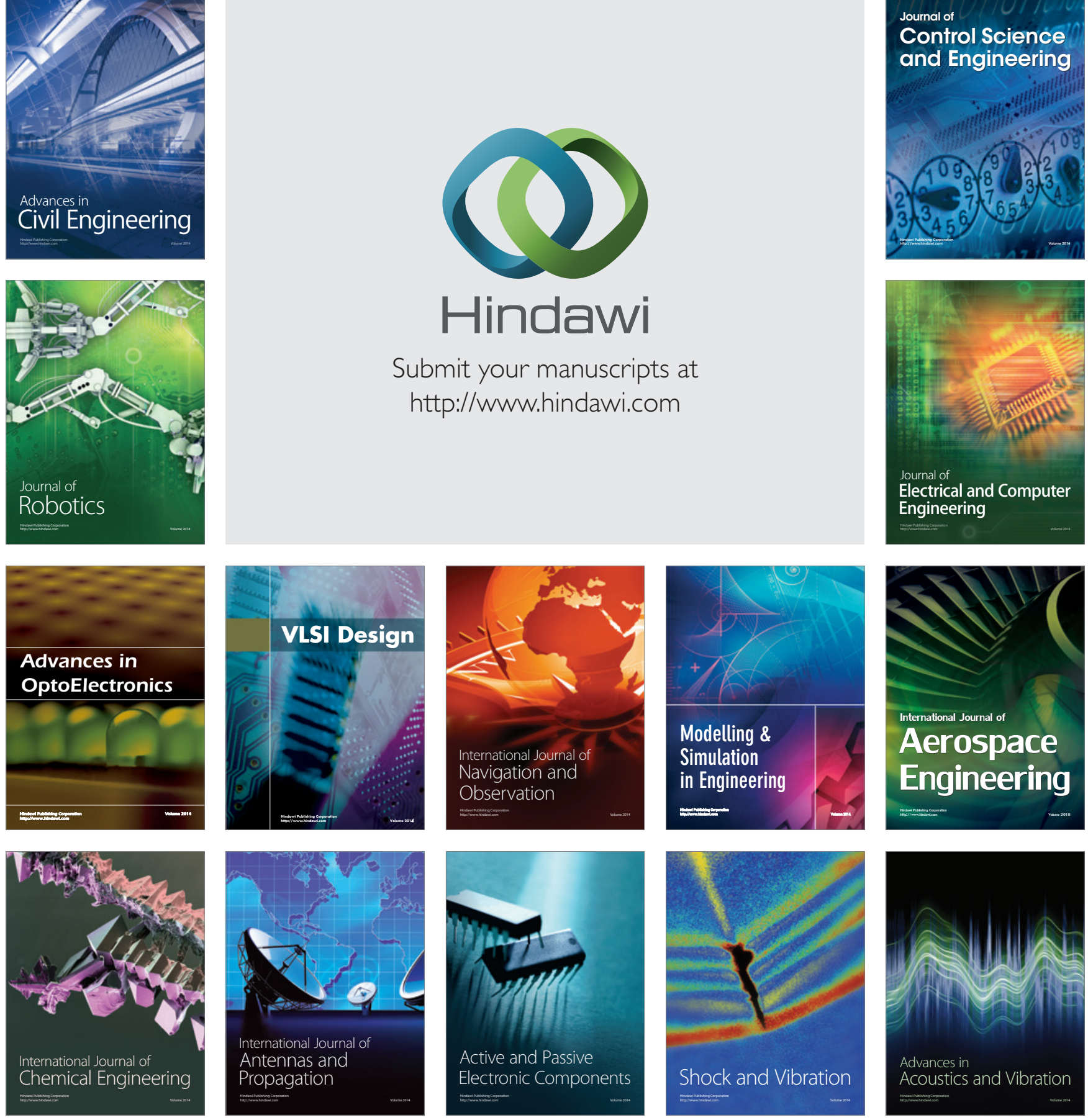